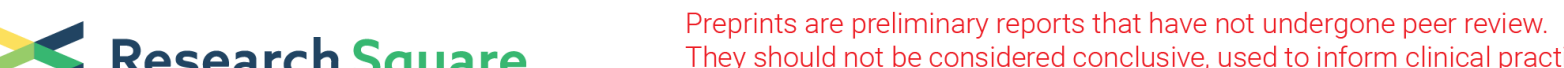 $\begin{array}{ll}\text { Research Square } & \text { They should not be considered conclusive, used to inform clinical practice, } \\ \text { or referenced by the media as validated information. }\end{array}$
}

\section{White Matter Tract Disruptions Predict Less Affected Hand Impairment Following Stroke: A Longitudinal Diffusion MRI Study}

Firdaus Fabrice Hannanu

University Grenoble Alpes: Universite Grenoble Alpes

\section{Bernadette Naegele}

Centre Hospitalier Universitaire Grenoble Alpes

Marc Hommel

University Grenoble Alpes: Universite Grenoble Alpes

Alexandre Krainik

Centre Hospitalier Universitaire Grenoble Alpes

Olivier Detante

Centre Hospitalier Universitaire Grenoble Alpes

Assia Jaillard ( $\nabla$ assia.jaillard@univ-grenoble-alpes.fr)

Centre Hospitalier Universitaire de Grenoble https://orcid.org/0000-0002-1045-671X

\section{Research Article}

Keywords: less-affected hand, ipsilateral pyramidal tract, contralesional hemisphere, sensorimotor systems, structural connectivity

Posted Date: November 12th, 2021

DOI: https://doi.org/10.21203/rs.3.rs-1050009/v1

License: (c) (i) This work is licensed under a Creative Commons Attribution 4.0 International License. Read Full License 


\section{Abstract}

Although less-affected hand ( $L A H)$ deficits following unilateral stroke are well documented, many aspects of LAH impairment mechanisms remain unresolved. To provide a better understanding of these mechanisms, we used diffusion MRI to examine the disruptions of white matter structural connections. Based on the redundancy theory, we hypothesized that a summation of motor-related tract disruptions would characterize LAH impairment.

We assessed LAH impairment and fractional anisotropy (FA) in 28 patients at one-month post-stroke (baseline), and 6 and 24 months later. LAH impairment was assessed with the Purdue Pegboard Test (PPT), handgrip strength, and movement time. FA was estimated in the CST, Anterior- Corona Radiata (ACR), and Limb of Internal Capsule (ALIC), Superior Longitudinal Fasciculus (SLF), and corpus callosum (CC). We used Linear Mixed Models to determine the tracts associated with LAH impairment over time.

Baseline PPT, grip, and movement time were impaired in $43 \%, 61 \%$, and $25 \%$, respectively. PPT was modeled by baseline ipsilesional-CST ( $t=3.75 ; p<0.001)$, ipsilesional-SLF $(t=3.19 ; p=0.002)$, contralesionalALIC ( $t=-4.89 ; p<0.001)$, and lesion volume $(t=-3.18 ; p=0.004)$; handgrip by baseline ipsilesional-CST $(t=3.39 ; p=0.001)$, contralesional-ALIC $(t=-3.91 ; p<0.001)$ and sex $(t=-1.43 ; p=0.007)$; movement time by baseline ipsilesional-SLF ( $t=-3.64 ; p=0.001), C C(t=4.00 ; p=<0.001)$, and lesion volume $(t=3.03 ; p=0.006)$.

In conclusion, white matter tract disruptions determine the LAH impairment profile, with ipsilesional-CST related to motor and ipsilesional-SLF to visuomotor processing. LAH impairment was associated with the summation of several tract disruptions, supporting the concept of cerebral redundancy. These results provide a theoretical basis for integrating LAH in rehabilitation programs and for treatment interventions such as neuromodulation.

\section{Introduction}

Hand movements represent a specific and essential function in humans required for everyday life activities. Following stroke, loss of hand functionality is one of the main factors affecting disability and remains a major target of rehabilitation interventions (Pennati et al., 2020; Pomeroy et al., 2011; Stinear, 2010). Parallel to sensorimotor deficits of the affected hand, the less-affected hand ( $L A H$, ipsilateral to the lesion) may show sensorimotor deficits for a large variety of sensorimotor tasks at the acute and chronic periods of stroke (Carey \& Matyas, 2011; Colebatch \& Gandevia, 1989; Gowers, 1886; Jones, Donaldson, \& Parkin, 1989; Kitsos, Hubbard, Kitsos, \& Parsons, 2013; Semrau et al., 2017; Varghese \& Winstein, 2019). LAH impairment is frequent (Semrau et al., 2017) and may compound functional disability and independence as patients require both hands to perform daily life activities (Plantin et al., 2021).

Although there are many unresolved aspects in our understanding of LAH impairment, several mechanisms are postulated (Kitsos et al., 2013). A first theory implicates the 'uncrossed' ipsilateral corticospinal tract (CST) (Ziemann et al., 1999) since 3-15\% of the corticospinal fibers descend in the 
ipsilateral spinal lateral funiculus without decussating in the medullary pyramids (Nyberg-Hansen, 1968; Schmahmann \& Pandya, 2006) (Fig. 1). Another theory relies on bilateral hemispheric control for unilateral movements (Chettouf, Rueda-Delgado, de Vries, Ritter, \& Daffertshofer, 2020; Jones et al., 1989; Kitsos et al., 2013; Noskin et al., 2008) as hand motor control is processed within the frontoparietal network including the superior longitudinal fasciculus (SLF) (Bundy \& Leuthardt, 2019). In addition, a role for the contralesional hemisphere has been reported based on studies showing activity imbalance between the sensorimotor cortices following stroke using fMRI (Dechaumont-Palacin et al., 2008; Favre et al., 2014; Rehme, Fink, von Cramon, \& Grefkes, 2011) or Transcranial Magnetic Stimulation (TMS) (Auriat, Neva, Peters, Ferris, \& Boyd, 2015). LAH impairment may also relate to interhemispheric transcallosal disconnections (Jung, Yoon, \& Park, 2002) as the corpus callosum (CC) coordinates motor function through the balance of excitatory and inhibitory interhemispheric interactions (Chettouf et al., 2020). Moreover, the impact of neuropsychological deficits such as apraxia and neglect has also been associated with LAH impairment (Chestnut \& Haaland, 2008; Sunderland, Bowers, Sluman, Wilcock, \& Ardron, 1999; Wetter, Poole, \& Haaland, 2005).

Anatomically, the main tracts engaged in hand sensorimotor control include the CST (Kuypers, 2011; Schmahmann \& Pandya, 2006), the body of the CC (CC-body) providing connections between the two sensorimotor cortices, the SLF for goal-directed actions and visuomotor processing (Budisavljevic et al., 2017; Schmahmann \& Pandya, 2006), and the cerebellar peduncles for movement coordination (Kelly \& Strick, 2003; Manto et al., 2012). In addition, the anterior corona radiata (ACR), anterior limb of the internal capsule (ALIC), and genu of the corpus callosum (genu-CC) carry fibers from the premotor, prefrontal, and orbitofrontal cortices projecting to the motor areas to provide motor information on movement preparation along with emotional and cognitive components of motor control (Fries, Danek, Scheidtmann, \& Hamburger, 1993; Morecraft et al., 2002; Schmahmann \& Pandya, 2006).

Based on the concept of cerebral redundancy (Glassman, 1987), we hypothesized that the combination of several mechanisms would yield LAH impairment, depending on the processes engaged in hand movements. Since diffusion MRI provides reliable measures of white matter microstructure such as fractional anisotropy (FA) reflecting the neural changes related to the stroke local lesion and its remote effects (Auriat et al., 2015; Kumar, Kathuria, Nair, \& Prasad, 2016; Lindenberg, Zhu, Ruber, \& Schlaug, 2012), we aimed to determine LAH impairment neural correlates using FA measures from the motorrelated tracts supporting hand movements. To this extent, we performed a longitudinal study assessing LAH impairment and FA at one month and 6 and 24-month follow-up in 28 patients with an ischemic stroke.

\section{Materials And Methods}

\subsection{Participants}

We enrolled 31 patients in the Randomized Controlled Stem Cell Trial (ISIS-HERMES) at the stroke unit of Grenoble Alpes University Hospital from October 2010 to 2014 (ClinicalTrials.gov NCT00875654). 
Patients received standard medical care including thrombolysis and thrombectomy when indicated. Patients were randomized to receive autologous mesenchymal stem cells (cell-therapy group) or rehabilitation alone (control group) in the first two weeks following stroke (Jaillard et al., 2020). Cell therapy was administered one month following stroke, after baseline clinical and MRI assessment. The ISIS-HERMES study was approved by the Institutional Review Board (CPP: 07-CHUG-25). Written informed consent was obtained from all patients before they participated in the study.

The main inclusion criteria were: age 18-70 years, first-ever unilateral infarct in the internal carotid artery territory, moderate to severe neurological deficit defined as NIHSS $\geq 7$, and the ability to follow a rehabilitation program. Exclusion criteria were pre-stroke neurological or psychiatric disease, severe medical condition, apraxia, or neglect diagnosed with an extinction NIHSS subscore $>1$. Complete criteria are provided in Table S1. Clinical, behavioral, and MRI measures were acquired at three sessions: onemonth post-stroke (M0), six months (M6), and 24 months (M24) follow-up (Fig S1).

\subsection{Demographic and Clinical Measures}

Age, sex, education level, height, weight, and stroke risk factors were recorded. Neurological severity was assessed using NIHSS and sensorimotor deficit using the Fugl-Meyer Score (FMS) (Fugl-Meyer, Jaasko, Leyman, Olsson, \& Steglind, 1975), with motor, sensory, and coordination subscores. A global cognitive assessment was performed with RBANS, exploring five domains (spatial, attention/executive, immediate and delayed memory, language) (Randolph, Tierney, Mohr, \& Chase, 1998). The global outcome was assessed using the Modified Rankin Score (mRS). (van Swieten, Koudstaal, Visser, Schouten, \& van Gijn, 1988). These assessments were performed by a stroke neurologist (NIHSS, mRS), neuropsychologists (RBANS, behavioral measures), and physiotherapists (FMS).

\subsection{Behavioral Measures}

We explored LAH impairment using three behavioral tests. The Purdue Pegboard Test (PPT) (Lafayette Instrument Company, Indiana)(Rapin, Tourk, \& Costa, 1966) was performed as described in http://www.equipement-ergotherapie.com/8-dexterité-manipulation.html, as a standardized quantitative test requiring motor (for grasping) and visuomotor (for reaching) components. The Hand dynamometer (Lafayette Instrument Company, Indiana; https://www.prohealthcareproducts.com/100-kg-220lb-handgrip-dynamometer-lafayette-instruments/) is a validated test to measure handgrip force (Grip). The Motor Screening Task (MST) measures movement time to assess sensorimotor deficits in CANTAB (https://www.cambridgecognition.com/cantab/cognitive-tests/attention/motor-screening-task-mot/). Raw scores were converted to percentiles to adjust for age and sex using published norms (Spreen \& Strauss, 1998) and CANTAB norms. Scores below the $5^{\text {th }}$ percentile were considered as impaired. The frequency of LAH impairment was also assessed in patients without cognitive deficit, defined as RBANS > 40 .

\subsection{MRI data acquisition}


The MRI protocol included structural and diffusion sequences. Patients were scanned at the IRMaGe MRI facility (Grenoble) on a 3T Philips magnet (Achieva 3.0 TTX; Philips, the Netherlands) with a 32-channel head coil. High resolution (1 mm3) sagittal 3D-T1-weighted (TR 9.9 ms, TE $4.6 \mathrm{~ms}$, flip angle $8^{\circ}$, TI 920 ms, inter shot time $1792 \mathrm{~ms}$ ) and fluid-attenuated inversion recovery (FLAIR) images (TR 8 s, TE 342 ms) were acquired. Diffusion-weighted images were acquired using single-shot echo-planar imaging (EPI) sequence (TR 11 milliseconds, TE 72 milliseconds, FOV 240 mm, slice thickness 2.0 mm, 70 axial slices, SENSE factor 2, fold-over direction anteroposterior, fat shift direction $\mathrm{P}$, fat suppression, and voxel size $1.67 * 1.67 * 2 \mathrm{~mm})$. We acquired 60 noncollinear directions with a b value of $1,000 \mathrm{~s} / \mathrm{mm}^{2}$ and 10 directions with a b value of $0 \mathrm{~s} / \mathrm{mm}^{2}$ that were averaged to give 1 average direction.

\subsection{MRI data analysis}

Structural images were used to manually delineate lesion masks and compute lesion volumes using MRIcron (https://www.nitrc.org/projects/mricron). Diffusion-weighted images were processed with the Diffusionist toolkit derived from FSL software, as previously described (Soulard et al., 2020). Each DWI image was visually checked and removed if corrupted. Then, after correction of eddy-current distortions, the diffusion tensor was estimated.

We used FA to assess white matter disruptions. Voxel-wise FA images were constructed from the resulting tensors. Linear and nonlinear registration transformations were applied to the FSL FA template in the MNI-152 space by incorporating the knowledge of each brain lesion using manually delineated lesion masks (Renard, Urvoy, \& Jaillard, 2015). FA was estimated only in the template's skeleton and outside the lesion mask. We estimated FA with atlas-based regions of interest (ROI) approach using the human brain white matter JHU atlas (Oishi et al., 2008) to analyse the descending motor tracts at different levels. Average FA values were estimated in 20 ROls listed in Table 2 and represented in Fig. S2. As we did not acquire sequences for susceptibility-induced distortions correction that are needed to obtain reliable diffusion values in the pons region, we excluded the pons ROI from our analysis. Diffusionist toolkit and related documentation can be found at http://mri-diffusionist.com/.

\subsection{Statistical analysis.}

LAH impairment was explored using descriptive statistics. To explore the mechanisms of LAH impairment, we first assessed the relationship between PPT, handgrip, and MST percentiles and clinical scores using Spearman correlations. Linear associations between ROI-derived FA and LAH raw scores were assessed using partial correlations controlling for age and sex, with bootstrap based on 1000 replications.

We used linear mixed models (LMMs) to determine which tracts (ROIs) influence LAH performances over time. The effects of ROIs, ROI by session interaction, lesion side, volume, handedness, education BMI, height, and weight were tested and included in the model only if significant. As cell therapy could have influenced outcomes, all LMMs were adjusted for cell therapy. Bonferroni correction was applied for post hoc multiple comparisons. Statistical significance was determined with the F-test $(p<0.05)$ and model fit 
was estimated with the Akaike Information Criterion (AIC) and by examining the distribution of residuals (Steyerberg et al., 2001). Model accuracy was assessed using $\mathrm{R}^{2}$. Statistical data analyses were performed using SPSS 23.0.

\section{Results}

Among 31 enrolled patients, 28 patients (20 males, 10 right lesions, 14 received cell therapy) completed clinical, behavioral, and MRI assessments at M0, M6, M24 (Fig. S1). Baseline clinical characteristics are presented in Table 1. Of note, the middle cerebral artery territory was involved in all patients (See lesion overlap, Fig. S3).

\subsection{LAH assessment}

At M0, LAH was impaired in 12 patients for PPT (43\%, 95\% Cl = 22-62\%), 17 for grip $(61 \%, 95 \% \mathrm{Cl}=42-$ $79 \%)$, and 4 for MST $(16 \%, 95 \% \mathrm{Cl}=3-31 \%)$. Then, performances improved over time until M24. While MST returned to normal values at M6, PPT and grip remained below the median (Fig. 2AC). In the subgroup of 20 patients without cognitive deficit, LAH at MO was impaired in $44 \%(95 \% \mathrm{Cl}=21-67)$ for PPT, $78 \%(95 \% \mathrm{Cl}=57-94)$ for handgrip, and $20 \%(95 \% \mathrm{Cl}=0-43)$ for MST (Fig. 2BD), with a similar temporal pattern to the whole group.

\subsection{Factors associated with LAH impairment}

At M0, PPT, Grip and MST significantly correlated with lesion volume and most clinical and cognitive scores (Table S2). LAH impairment was strongly correlated with mRS at M6 and M24 (Table S3). There was no significant effect of lesion side on LAH performances.

LAH and FA correlations are presented in Table 2. PPT correlated with all ipsilesional (i-) CST ROIs, contralesional (C-) CP, and bilateral SLF, corpus callosum, ALIC, and ACR. Grip correlated with the same ROls except for i-SLF and i-ALIC. MST correlated with i-PLIC, i-CP, C-SLF, genu-CC, bilateral ACR, and cALIC. There were no significant correlations with the cerebellar peduncles.

LMMs results are presented in Table 3 and S4-S6. PPT performance over time was predicted by i-PLIC $(t=3.75 ; p<0.001), i-S L F(t=3.19 ; p=0.002)$, and $c-A L I C(t=-4.89 ; p<0.001)$ at M0, with a significant effect of lesion-volume ( $t=-3.18 ; p=0.004)$, but no age, sex or cell-therapy effect. Model accuracy was good $\left(r^{2}=0.831\right)$. Grip performance was modeled by $\mathrm{i}-\mathrm{CP}(\mathrm{t}=3.39 ; \mathrm{p}=0.001)$ and $\mathrm{c}-\mathrm{ALIC}(\mathrm{t}=-3.91 ; \mathrm{p}<0.001)$ at M0 and male sex $(t=3.05 ; p=0.007)$, with no effect of age and cell-therapy. Model accuracy was good $\left(r^{2}=0.849\right)$. Longer MST was modeled by $i-S L F$ at M0 $(t=-3.64 ; p=0.001), M 6(t=-3.52 ; p=0.001)$, and M24 $(t=-3.31 ; p=0.003), C C$-body at $M 0(t=4.00 ; p=<0.001)$ and $M 6(t=3.08 ; p=0.005)$, and volume $(t=3.03$; $p=0.006)$, with no effect of session, age or sex. Model accuracy was excellent $\left(r^{2}=0.978\right)$.

\section{Discussion}




\subsection{Clinical assessment of LAH}

In this longitudinal study, we assessed behavioral performances of the less-affected hand (LAH) in 28 patients at three sessions. LAH impairment was frequent one-month post-stroke, ranging from $16-61 \%$, in line with previous studies. (Jones et al., 1989; Kitsos et al., 2013; Marque et al., 1997; Noskin et al., 2008; Semrau et al., 2017) At the chronic phase of stroke, the impairment of LAH was influenced by the type of the task. PPT and handgrip that require motor components remained impaired, while MST may be insensitive to movement time delays that require kinematic measures to be evidenced (Bustren, Sunnerhagen, \& Alt Murphy, 2017; Metrot et al., 2013). Furthermore, baseline LAH performances correlated with the outcome at follow-ups and may become a useful tool in future clinical trials.

\subsection{Mechanisms of LAH impairment}

Our findings showed that several tracts including the i-CST, c-ALIC, CC, and i-SLF, depending on the task, were associated with LAH impairment.

We found moderate to strong correlations between the three LAH scores and FA in the ipsilesional CST, while no correlation was observed with contralesional CST-CSR and CST-PLIC, suggesting that LAH impairment is driven by the ipsilesional CST. Furthermore, i-CST was a strong predictor of PPT and grip impairment. These findings suggest that LAH impairment requiring motor processing such as PPT and grip relates to the damaged uncrossed fibers of the ipsilesional CST (Fig. 1 \& S4A). These fibers terminate in the ventromedial intermediate zone to propriospinal neurons connected to distal motoneurons through intersegment spinal interneurons and may be involved in the motor control of dexterous hand movements (Tohyama et al., 2017). Taken together, our results support the hypothesis that damaged uncrossed ipsilateral CST fibers contribute to the motor components of LAH impairment.

LAH scores were also correlated with FA in bilateral ALIC and ACR, and CC. LMMs showed that PPT and handgrip impairment was associated with c-ALIC. Although ACR has been linked to cognition and particularly to attention in adults with brain injury (Niogi et al., 2008), a part of ACR fibers originate in the SMA, descend through the ALIC (Morecraft et al., 2002), and then merge with the CST in the CP (Schmahmann \& Pandya, 2006), which continues in the pons and medulla to decussate at the pyramid caudal end (Fries et al., 1993; Schmahmann \& Pandya, 2006). The involvement of SMA in simple motor tasks is documented by stroke studies, with SMA lesions leading to mild motor deficits (Fries et al., 1993), and i-SMA fMRI-related activity supporting motor recovery (Favre et al., 2014; Grefkes et al., 2008). Therefore, motor control components of LAH impairment may also implicate the contralesional CST through the transcallosal fibers and C-ALIC fibers from premotor and/or prefrontal areas (Fig. 1 and S4.B).

We found that LAH scores correlated with CC FA, which predicted MST. A role of the CC is motor coordination of bimanual (Andres et al., 1999) and unilateral hand motor movements through the balance of excitatory and inhibitory interhemispheric interactions (Chettouf et al., 2020). The ipsi- and contralesional motor areas exert a reciprocal influence through transcallosal fibers (Leichnetz, 1986), as evidenced in tracer studies showing reciprocal transcallosal connections for both MI and SMA (Gould, 
Cusick, Pons, \& Kaas, 1986). In nonhuman primates, SMA lesions impaired the LAH motor program through transcallosal connections to contralesional SMA (Brinkman, 1984). Of note, c-ALIC and CC predicted worse LAH performances when accounting for the effects of i-CST and i-SLF, suggesting that LAH impairment may be compounded by the imbalance between ipsilesional and contralesional motor regions.

Our results showed that LAH impairment correlated with decreased FA in bilateral SLF and that i-SLF predicted PPT and MST. These findings support the theory that LAH impairment relates to unilateral movement bilateral hemispheric control (Chettouf et al., 2020; Jones et al., 1989; Kitsos et al., 2013; Noskin et al., 2008). In this view, the damaged hemisphere would alter LAH movements. Indeed, unimanual motor tasks implicating visuomotor components yield bilateral activity in the frontoparietal network (Cavina-Pratesi et al., 2018; Cavina-Pratesi et al., 2010; Chettouf et al., 2020; Culham et al., 2003). In agreement with this literature, PPT and MST, which require visuomotor control in contrast to handgrip, were associated with the SLF, a key structure of the frontoparietal network connecting parietal, premotor, and motor frontal areas in both human (Makris et al., 2005; Thiebaut de Schotten et al., 2011; Wang et al., 2016) and nonhuman primates (Schmahmann \& Pandya, 2006). Furthermore, our findings that SLF disruptions alter LAH with visuomotor processing are supported by previous works showing an essential role for the SLF in motor planning and kinematic components of movement execution (Budisavljevic et al., 2017),.

We found consistent correlations between LAH impairment and spatial and attentional cognitive deficits that may result from SLF, ACR or ALIC disruptions, depending on the cognitive domain. Interestingly, excluding patients with cognitive deficit did not significantly improve LAH performances, suggesting that cognitive impairment was not a major cause of LAH impairment in this study. Nevertheless, as patients with severe apraxia or neglect were excluded from our study, we may have underestimated the influence of cognitive impairment on LAH impairment related to apraxia and neglect (Sunderland et al., 1999).

\subsection{The role of the lesion volume}

Lesion volume correlated with LAH impairment and predicted PPT and MST, consistently with nonhuman macaque experiments (Darling et al., 2011). Surprisingly, few studies, if any, have explored the relationships between lesion volume and LAH impairment in humans. Our results showing that LAH tasks with visuomotor processing were compounded by lesion volume provide empirical support for the notion of mass action through redundancy in the visuomotor system (Glassman, 1987).

\subsection{Limitations}

The small sample size is the main limitation of this study. However, this is the first study exploring the microstructural white matter disruptions to understand the underlying mechanisms of LAH impairment following stroke. Also, the longitudinal design with repeated measures for both behavioral and FA measures, the homogeneity of our population in terms of age, absence of leukoaraïosis, and stroke severity and territory, and the high model accuracy suggest that the sample size was adequate for this 
study. Nevertheless, the small sample may explain why we did not observe any effect of the lesion side, in contrast with others (Varghese \& Winstein, 2019). Moreover, this study was part of a randomized clinical trial assessing cell therapy, which might have influenced outcomes. To account for this limitation, all LMMs were adjusted for cell therapy.

\section{Conclusion}

This study showed that motor-related tract disruptions predict LAH impairment, with a pattern that varies according to the motor and visuomotor processing: tasks with motor processing were associated with the ipsilateral CST implicating the involvement of uncrossed CST fibers, while tasks with visuomotor processing were related to the SLF supporting hand motor control and to lesion volume. In addition, the contralesional hemisphere may play a role in LAH impairment by influencing the planning and execution of hand movements through prefrontal/premotor areas and transcallosal interactions. Taken together, our findings suggest that LAH impairment requires the summation of tract disruptions, supporting the concept of cerebral redundancy for the motor system. Our results provide a theoretical basis for integrating LAH impairment in rehabilitation programs to improve functional recovery and for research interventions, such as neuromodulation.

\section{Glossary}

General: FA= fraction of Anisotropy (diffusion MRI measure of white matter integrity); FMS: Fugl-Meyer Score (motor-FMS subscore from 0 to 100 for sensorimotor functions; IQR: Interquartile Range; LAH= Less-Affected Hand; MST: Movement Screening Test = time; NIHSS: National Institute of Health Stroke Scale (neurological severity); PPT: Purdue Pegboard Test; RBANS: Repeatable Battery for the Assessment of Neuropsychological Status (global assessment of cognitive functions with a mean of 100 in healthy participants); SD: Standard deviation;TMS= Transcranial Magnetic Stimulation.

White matter tracts and Regions of Interest (ROIs): ACR= Anterior Corona Radiata; ALIC= Anterior Limb of Internal Capsule; body-CC= body or middle segment of the Corpus Callosum (CC3 and CC4); CST=Corticospinal tract (including SCR= Superior Corona Radiata; PLIC= Posterior Limb of Internal Capsule; $\mathrm{CP}=$ Cerebral Peduncle); $\mathrm{CRP}=$ CorticoReticular Pathway; genu-CC= genu of the Corpus Callosum; $\mathrm{ICP}=$ inferior cerebellar peduncle; $\mathrm{MCP}=$ middle cerebellar peduncle; $\mathrm{SCP}=$ superior cerebellar peduncle; $\mathrm{SLF}=$ superior longitudinal fasciculus; $\mathrm{i}=\mathrm{ipsilesional}$ and $\mathrm{c}=$ contralesional tracts.

\section{Declarations}

\section{Data availability statement}

The data that support the findings of this study are available from the corresponding author upon reasonable request criteria.

\section{Ethical approval}


Institutional review board approval and written informed consent from all patients or relatives were obtained. Data are reported according to the STROBE guidelines.

\section{Declaration of competing interest}

The authors have no conflict of interest to declare.

\section{Acknowledgements}

We thank the platform of France Life Imaging network for partly supporting MRI data acquisition through the grant "ANR-11-INBS-0006, the Clinical Investigation Center (CIC) INSERM UMS 002 CHU Grenoble Alpes for data monitoring, and Felix Renard and Bérengère Aubert-Broche for the Diffusionist software (Diffusion MRI processing).

\section{Author contributions}

F.F.H performed the data processing, statistical analysis, prepared the visualization and wrote the manuscript. B.N. was involved in study conceptualization and data collection. A.K was involved in funding acquisition, study conceptualization, and supervision. O.D was involved in funding acquisition, study conceptualization, data collection, and project administration. A.J was involved in funding acquisition, study conceptualization, data processing, statistical analysis, project administration, supervision, and wrote the manuscript. All authors critically reviewed the manuscript.

\section{Funding sources}

This work was supported by: Ministère des Solidarités et de la Santé. Programme hospitalier de recherche clinique - PHRC: ISIS-2007PHR04 (NCT00875654) and HERMES-2007-A00853-50 for patient inclusion and clinical and multimodal MRI data acquisition; IRMaGE platform partly funded by the French program "investissement d'avenir" run by the agence nationale pour la recherche, grant " infrastructure d'avenir en Biologie Santé » - ANR - 11 - INBS-0006m; and the Indonesia Endowment Fund for Education (LPDP) for F. F. Hannanu PhD.

\section{References}

1. Andres, F. G., Mima, T., Schulman, A. E., Dichgans, J., Hallett, M., \& Gerloff, C. (1999). Functional coupling of human cortical sensorimotor areas during bimanual skill acquisition. Brain, 122 (Pt 5), 855-870. doi: 10.1093/brain/122.5.855

2. Auriat, Angela M., Neva, Jason L., Peters, Sue, Ferris, Jennifer K., \& Boyd, Lara A. (2015). A Review of Transcranial Magnetic Stimulation and Multimodal Neuroimaging to Characterize Post-Stroke Neuroplasticity. Frontiers in neurology, 6, 226-226. doi: 10.3389/fneur.2015.00226

3. Brinkman, C. (1984). Supplementary motor area of the monkey's cerebral cortex: short- and long-term deficits after unilateral ablation and the effects of subsequent callosal section. $J$ Neurosci, 4(4), 918- 
929.

4. Budisavljevic, S., Dell'Acqua, F., Zanatto, D., Begliomini, C., Miotto, D., Motta, R., \& Castiello, U. (2017). Asymmetry and Structure of the Fronto-Parietal Networks Underlie Visuomotor Processing in Humans. Cereb Cortex, 27(2), 1532-1544. doi: 10.1093/cercor/bhv348

5. Bundy, D. T., \& Leuthardt, E. C. (2019). The Cortical Physiology of Ipsilateral Limb Movements. Trends Neurosci, 42(11), 825-839. doi: 10.1016/j.tins.2019.08.008

6. Bustren, E. L., Sunnerhagen, K. S., \& Alt Murphy, M. (2017). Movement Kinematics of the Ipsilesional Upper Extremity in Persons With Moderate or Mild Stroke. Neurorehabil Neural Repair, 31(4), 376-386. doi: $10.1177 / 1545968316688798$

7. Carey, L. M., \& Matyas, T. A. (2011). Frequency of discriminative sensory loss in the hand after stroke in a rehabilitation setting. J Rehabil Med, 43(3), 257-263. doi: 10.2340/16501977-0662

8. Cavina-Pratesi, C., Connolly, J. D., Monaco, S., Figley, T. D., Milner, A. D., Schenk, T., \& Culham, J. C. (2018). Human neuroimaging reveals the subcomponents of grasping, reaching and pointing actions. Cortex, 98, 128-148. doi: 10.1016/j.cortex.2017.05.018

9. Cavina-Pratesi, C., Monaco, S., Fattori, P., Galletti, C., McAdam, T. D., Quinlan, D. J., ... Culham, J. C. (2010). Functional magnetic resonance imaging reveals the neural substrates of arm transport and grip formation in reach-to-grasp actions in humans. J Neurosci, 30(31), 10306-10323. doi: 10.1523/JNEUROSCI.2023-10.2010

10. Chestnut, C., \& Haaland, K. Y. (2008). Functional significance of ipsilesional motor deficits after unilateral stroke. Arch Phys Med Rehabil, 89(1), 62-68. doi: 10.1016/j.apmr.2007.08.125

11. Chettouf, S., Rueda-Delgado, L. M., de Vries, R., Ritter, P., \& Daffertshofer, A. (2020). Are unimanual movements bilateral? Neurosci Biobehav Rev, 113, 39-50. doi: 10.1016/j.neubiorev.2020.03.002

12. Colebatch, J. G., \& Gandevia, S. C. (1989). The distribution of muscular weakness in upper motor neuron lesions affecting the arm. Brain, 112 (Pt 3), 749-763. doi: 10.1093/brain/112.3.749

13. Culham, J. C., Danckert, S. L., DeSouza, J. F., Gati, J. S., Menon, R. S., \& Goodale, M. A. (2003). Visually guided grasping produces $\mathrm{fMRI}$ activation in dorsal but not ventral stream brain areas. Exp Brain Res, 153(2), 180-189. doi: 10.1007/s00221-003-1591-5

14. Darling, W. G., Pizzimenti, M. A., Hynes, S. M., Rotella, D. L., Headley, G., Ge, J., . . Morecraft, R. J. (2011). Volumetric effects of motor cortex injury on recovery of ipsilesional dexterous movements. Exp Neurol, 231(1), 56-71. doi: 10.1016/j.expneurol.2011.05.015

15. Dechaumont-Palacin, S., Marque, P., De Boissezon, X., Castel-Lacanal, E., Carel, C., Berry, I., . . . Loubinoux, I. (2008). Neural correlates of proprioceptive integration in the contralesional hemisphere of very impaired patients shortly after a subcortical stroke: an FMRI study. Neurorehabil Neural Repair, 22(2), 154-165. doi: 10.1177/1545968307307118

16. Favre, I., Zeffiro, T. A., Detante, O., Krainik, A., Hommel, M., \& Jaillard, A. (2014). Upper limb recovery after stroke is associated with ipsilesional primary motor cortical activity: a meta-analysis. Stroke, 45(4), 1077-1083. doi: 10.1161/STROKEAHA.113.003168 
17. Fries, W., Danek, A., Scheidtmann, K., \& Hamburger, C. (1993). Motor recovery following capsular stroke. Role of descending pathways from multiple motor areas. Brain, 116 ( Pt 2), 369-382. doi: 10.1093/brain/116.2.369

18. Fugl-Meyer, A. R., Jaasko, L., Leyman, I., Olsson, S., \& Steglind, S. (1975). The post-stroke hemiplegic patient. 1. a method for evaluation of physical performance. Scand J Rehabil Med, 7(1), 13-31.

19. Glassman, R. B. (1987). An hypothesis about redundancy and reliability in the brains of higher species: analogies with genes, internal organs, and engineering systems. Neurosci Biobehav Rev, 11(3), 275-285. doi: 10.1016/s0149-7634(87)80014-3

20. Gould, H. J., 3rd, Cusick, C. G., Pons, T. P., \& Kaas, J. H. (1986). The relationship of corpus callosum connections to electrical stimulation maps of motor, supplementary motor, and the frontal eye fields in owl monkeys. J Comp Neurol, 247(3), 297-325. doi: 10.1002/cne.902470303

21. Gowers, W.R. (1886). A Manual of Diseases of the Nervous System. Motor Symptoms. (Vol. II). London UK: Churchill.

22. Grefkes, C., Nowak, D. A., Eickhoff, S. B., Dafotakis, M., Kust, J., Karbe, H., \& Fink, G. R. (2008). Cortical connectivity after subcortical stroke assessed with functional magnetic resonance imaging. Ann Neurol, 63(2), 236-246. doi: 10.1002/ana.21228

23. Jaillard, A., Hommel, M., Moisan, A., Zeffiro, T. A., Favre-Wiki, I. M., Barbieux-Guillot, M., . . Detante, O. (2020). Autologous Mesenchymal Stem Cells Improve Motor Recovery in Subacute Ischemic Stroke: a Randomized Clinical Trial. Trans/ Stroke Res. doi: 10.1007/s12975-020-00787-z

24. Jones, R. D., Donaldson, I. M., \& Parkin, P. J. (1989). Impairment and recovery of ipsilateral sensorymotor function following unilateral cerebral infarction. Brain, 112 (Pt 1), 113-132. doi: 10.1093/brain/112.1.113

25. Jung, H. Y., Yoon, J. S., \& Park, B. S. (2002). Recovery of proximal and distal arm weakness in the ipsilateral upper limb after stroke. NeuroRehabilitation, 17(2), 153-159.

26. Kelly, R. M., \& Strick, P. L. (2003). Cerebellar loops with motor cortex and prefrontal cortex of a nonhuman primate. J Neurosci, 23(23), 8432-8444. doi: 10.1523/jneurosci.23-23-08432.2003

27. Kitsos, G. H., Hubbard, I. J., Kitsos, A. R., \& Parsons, M. W. (2013). The ipsilesional upper limb can be affected following stroke. ScientificWorldJournal, 2013, 684860. doi: 10.1155/2013/684860

28. Kumar, P., Kathuria, P., Nair, P., \& Prasad, K. (2016). Prediction of Upper Limb Motor Recovery after Subacute Ischemic Stroke Using Diffusion Tensor Imaging: A Systematic Review and Meta-Analysis. J Stroke, 18(1), 50-59. doi: 10.5853/jos.2015.01186

29. Kuypers, H. (2011). Anatomy of the Descending Pathways (Vol. II).

30. Leichnetz, G. R. (1986). Afferent and efferent connections of the dorsolateral precentral gyrus (area 4, hand/arm region) in the macaque monkey, with comparisons to area 8. J Comp Neurol, 254(4), 460492. doi: $10.1002 /$ cne. 902540403

31. Lindenberg, R., Zhu, L. L., Ruber, T., \& Schlaug, G. (2012). Predicting functional motor potential in chronic stroke patients using diffusion tensor imaging. Hum Brain Mapp, 33(5), 1040-1051. doi: $10.1002 / \mathrm{hbm} .21266$ 
32. Makris, N., Kennedy, D. N., Mclnerney, S., Sorensen, A. G., Wang, R., Caviness, V. S., Jr., \& Pandya, D. N. (2005). Segmentation of subcomponents within the superior longitudinal fascicle in humans: a quantitative, in vivo, DT-MRI study. Cereb Cortex, 15(6), 854-869. doi: 10.1093/cercor/bhh186

33. Manto, Mario, Bower, James M., Conforto, Adriana Bastos, Delgado-García, José M., da Guarda, Suzete Nascimento Farias, Gerwig, Marcus, . . Timmann, Dagmar. (2012). Consensus paper: roles of the cerebellum in motor control-the diversity of ideas on cerebellar involvement in movement. Cerebellum (London, England), 11(2), 457-487. doi: 10.1007/s12311-011-0331-9

34. Marque, P., Felez, A., Puel, M., Demonet, J. F., Guiraud-Chaumeil, B., Roques, C. F., \& Chollet, F. (1997). Impairment and recovery of left motor function in patients with right hemiplegia. $J$ Neurol Neurosurg Psychiatry, 62(1), 77-81. doi: 10.1136/jnnp.62.1.77

35. Metrot, J., Froger, J., Hauret, I., Mottet, D., van Dokkum, L., \& Laffont, I. (2013). Motor recovery of the ipsilesional upper limb in subacute stroke. Arch Phys Med Rehabil, 94(11), 2283-2290. doi: 10.1016/j.apmr.2013.05.024

36. Morecraft, R. J., Herrick, J. L., Stilwell-Morecraft, K. S., Louie, J. L., Schroeder, C. M., Ottenbacher, J. G., \& Schoolfield, M. W. (2002). Localization of arm representation in the corona radiata and internal capsule in the non-human primate. Brain, 125(Pt 1), 176-198. doi: 10.1093/brain/awf011

37. Niogi, S. N., Mukherjee, P., Ghajar, J., Johnson, C. E., Kolster, R., Lee, H., . . McCandliss, B. D. (2008). Structural dissociation of attentional control and memory in adults with and without mild traumatic brain injury. Brain, 131(Pt 12), 3209-3221. doi: 10.1093/brain/awn247

38. Noskin, O., Krakauer, J. W., Lazar, R. M., Festa, J. R., Handy, C., O'Brien, K. A., \& Marshall, R. S. (2008). Ipsilateral motor dysfunction from unilateral stroke: implications for the functional neuroanatomy of hemiparesis. J Neurol Neurosurg Psychiatry, 79(4), 401-406. doi: 10.1136/jnnp.2007.118463

39. Nyberg-Hansen, R. (1968). [Functional-anatomical aspects of the gamma-motor neurons and their afferent connections]. Tidsskr Nor Laegeforen, 88(5), 319-325.

40. Oishi, K., Zilles, K., Amunts, K., Faria, A., Jiang, H., Li, X., . . Mori, S. (2008). Human brain white matter atlas: identification and assignment of common anatomical structures in superficial white matter. Neuroimage, 43(3), 447-457. doi: 10.1016/j.neuroimage.2008.07.009

41. Pennati, G. V., Plantin, J., Carment, L., Roca, P., Baron, J. C., Pavlova, E., . . Lindberg, P. G. (2020). Recovery and Prediction of Dynamic Precision Grip Force Control After Stroke. Stroke, 51(3), 944-951. doi: 10.1161/STROKEAHA.119.026205

42. Plantin, J., Verneau, M., Godbolt, A. K., Pennati, G. V., Laurencikas, E., Johansson, B., .. . Lindberg, P. G. (2021). Recovery and Prediction of Bimanual Hand Use After Stroke. Neurology, 97(7), e706-e719. doi: 10.1212/WNL.0000000000012366

43. Pomeroy, V., Aglioti, S. M., Mark, V. W., McFarland, D., Stinear, C., Wolf, S. L., ... Fitzpatrick, S. M. (2011). Neurological principles and rehabilitation of action disorders: rehabilitation interventions. Neurorehabil Neural Repair, 25(5 Suppl), 33S-43S. doi: 10.1177/1545968311410942

44. Randolph, C., Tierney, M. C., Mohr, E., \& Chase, T. N. (1998). The Repeatable Battery for the Assessment of Neuropsychological Status (RBANS): preliminary clinical validity. J Clin Exp 
Neuropsychol, 20(3), 310-319. doi: 10.1076/jcen.20.3.310.823

45. Rapin, I., Tourk, L. M., \& Costa, L. D. (1966). Evaluation of the Purdue Pegboard as a screening test for brain damage. Dev Med Child Neurol, 8(1), 45-54.

46. Rehme, A. K., Fink, G. R., von Cramon, D. Y., \& Grefkes, C. (2011). The role of the contralesional motor cortex for motor recovery in the early days after stroke assessed with longitudinal FMRI. Cereb Cortex, 21(4), 756-768. doi: 10.1093/cercor/bhq140

47. Renard, Félix, Urvoy, Matthieu, \& Jaillard, Assia. (2015). Bayesian Stroke Lesion Estimation for Automatic Registration of DTI Images. https://doi.org/10.1007/978-3-319-30858-6_9

48. Schmahmann, J.D., \& Pandya, D.N. (2006). Fiber pathways of the brain. Oxford: Oxford University Press.

49. Semrau, J. A., Herter, T. M., Kenzie, J. M., Findlater, S. E., Scott, S. H., \& Dukelow, S. P. (2017). Robotic Characterization of Ipsilesional Motor Function in Subacute Stroke. Neurorehabil Neural Repair, 31(6), 571-582. doi: 10.1177/1545968317704903

50. Soulard, J., Huber, C., Baillieul, S., Thuriot, A., Renard, F., Aubert Broche, B., . . Group, Isis-Hermes. (2020). Motor tract integrity predicts walking recovery: A diffusion MRI study in subacute stroke. Neurology, 94(6), e583-e593. doi: 10.1212/WNL.0000000000008755

51. Spreen, O., \& Strauss, E. . (1998). A compendium of neuropsychological test (Second ed.). New York, USA: Oxford University Press.

52. Steyerberg, E. W., Harrell, F. E., Jr., Borsboom, G. J., Eijkemans, M. J., Vergouwe, Y., \& Habbema, J. D. (2001). Internal validation of predictive models: efficiency of some procedures for logistic regression analysis. J Clin Epidemiol, 54(8), 774-781.

53. Stinear, C. (2010). Prediction of recovery of motor function after stroke. Lancet Neurol, 9(12), 12281232. doi: 10.1016/S1474-4422(10)70247-7

54. Sunderland, A., Bowers, M. P., Sluman, S. M., Wilcock, D. J., \& Ardron, M. E. (1999). Impaired dexterity of the ipsilateral hand after stroke and the relationship to cognitive deficit. Stroke, 30(5), 949-955. doi: 10.1161/01.str.30.5.949

55. Thiebaut de Schotten, M., Dell'Acqua, F., Forkel, S. J., Simmons, A., Vergani, F., Murphy, D. G., \& Catani, M. (2011). A lateralized brain network for visuospatial attention. Nat Neurosci, 14(10), 1245-1246. doi: $10.1038 / \mathrm{nn} .2905$

56. van Swieten, J. C., Koudstaal, P. J., Visser, M. C., Schouten, H. J., \& van Gijn, J. (1988). Interobserver agreement for the assessment of handicap in stroke patients. Stroke, 19(5), 604-607. doi:

10.1161/01.str.19.5.604

57. Varghese, R., \& Winstein, C. J. (2019). Relationship Between Motor Capacity of the Contralesional and Ipsilesional Hand Depends on the Side of Stroke in Chronic Stroke Survivors With Mild-toModerate Impairment. Front Neurol, 10, 1340. doi: 10.3389/fneur.2019.01340

58. Wang, X., Pathak, S., Stefaneanu, L., Yeh, F. C., Li, S., \& Fernandez-Miranda, J. C. (2016). Subcomponents and connectivity of the superior longitudinal fasciculus in the human brain. Brain Struct Funct, 221(4), 2075-2092. doi: 10.1007/s00429-015-1028-5 
59. Wetter, S., Poole, J. L., \& Haaland, K. Y. (2005). Functional implications of ipsilesional motor deficits after unilateral stroke. Arch Phys Med Rehabil, 86(4), 776-781. doi: 10.1016/j.apmr.2004.08.009

60. Ziemann, U., Ishii, K., Borgheresi, A., Yaseen, Z., Battaglia, F., Hallett, M., . . Wassermann, E. M. (1999). Dissociation of the pathways mediating ipsilateral and contralateral motor-evoked potentials in human hand and arm muscles. J Physiol, 518 (Pt 3), 895-906. doi: 10.1111/j.14697793.1999.0895p.x

\section{Tables}

Table 1. Baseline clinical characteristics

\begin{tabular}{|lllll|}
\hline Characteristics & Mean & SD & Median & IQR \\
\hline Age & 53.32 & 9.41 & 55 & 13 \\
\hline Lesion Volume $\left(\mathrm{cm}^{3}\right)$ & 104.93 & 66.28 & 90 & 115 \\
\hline NIHSS & 14.07 & 4.87 & 12 & 7 \\
\hline motor NIHSS & 6.29 & 2.9 & 7 & 4 \\
\hline FMS & 135 & 40.06 & 129 & 61 \\
\hline motor FMS & 35.61 & 27.98 & 29 & 36 \\
\hline coordination FMS & 7.64 & 4.28 & 9 & 6 \\
\hline sensory FMS & 11.14 & 8.01 & 14 & 17 \\
\hline RBANS & 53.93 & 25.83 & 55 & 49 \\
\hline
\end{tabular}

Table 2. Partial correlation coefficients adjusted for age and sex between behavioral scores and FA at Mo. 


\begin{tabular}{llllll} 
PPT & \multicolumn{3}{c}{ Grip } & \multicolumn{3}{c}{ MST } \\
$R$ & $95 \% \mathrm{Cl}$ & $R$ & $95 \% \mathrm{Cl}$ & $R$ & $95 \% \mathrm{Cl}$ \\
& [lower, upper] & & [lower, upper] & & [lower, upper]
\end{tabular}

Corticospinal tract FA

\begin{tabular}{lllllll} 
i-CST-SCR & $0.479^{*}$ & {$[0.193,0.750]$} & $0.441^{*}$ & {$[0.018,0.747]$} & -0.369 & {$[-0.688,0.048]$} \\
\hline C-CST-SCR & 0.174 & {$[-0.187,0.509]$} & 0.009 & {$[-0.413,0.563]$} & -0.154 & {$[-0.524,0.214]$} \\
\hline i-CST-PLIC & $0.583^{\star}$ & {$[0.296,0.797]$} & $0.456^{*}$ & {$[0.076,0.732]$} & $-0.433^{\star}$ & {$[-0.715,-0.044]$} \\
\hline c-CST-PLIC & 0.015 & {$[-0.416,0.452]$} & 0.009 & {$[-0.385,0.460]$} & 0.023 & {$[-0.450,0.433]$} \\
\hline i-CST-CP & $0.548^{\star}$ & {$[0.228,0.805]$} & $0.563^{*}$ & {$[0.173,0.808]$} & $-0.426^{*}$ & {$[-0.727,-0.014]$} \\
\hline c-CST-CP & $0.475^{\star}$ & {$[0.092,0.714]$} & $0.461^{\star}$ & {$[0.036,0.713]$} & -0.387 & {$[-0.677,0.095]$}
\end{tabular}

Cerebral tract FA

\begin{tabular}{lllllll}
\hline i-SLF & $0.490^{*}$ & {$[0.043,0.803]$} & 0.412 & {$[-0.147,0.778]$} & $-0.528^{*}$ & {$[-0.792,-0.135]$} \\
\hline C-SLF & $0.458^{*}$ & {$[0.056,0.734]$} & $0.478^{*}$ & {$[0.246,0.736]$} & $-0.504^{*}$ & {$[-0.77,-0.152]$} \\
\hline Body-CC & $0.485^{*}$ & {$[0.180,0.787]$} & $0.510^{*}$ & {$[0.113,0.804]$} & -0.393 & {$[-0.698,-0.027]$} \\
\hline Genu-CC & $0.581^{*}$ & {$[0.251,0.883]$} & $0.665^{*}$ & {$[0.370,0.862]$} & $-0.658^{*}$ & {$[-0.923,-0.316]$} \\
\hline i-ACR & $0.550^{*}$ & {$[0.248,0.808]$} & $0.527^{*}$ & {$[0.150,0.834]$} & $-0.575^{*}$ & {$[-0.809,-0.263]$} \\
\hline c-ACR & $0.538^{*}$ & {$[0.189,0.809]$} & $0.678^{*}$ & {$[0.449,0.84]$} & $-0.591^{*}$ & {$[-0.851,-0.204]$} \\
\hline i-ALIC & $0.483^{*}$ & {$[0.209,0.743]$} & 0.368 & {$[0.003,0.658]$} & -0.337 & {$[-0.684,0.071]$} \\
\hline C-ALIC & $0.454^{*}$ & {$[0.007,0.773]$} & $0.540^{*}$ & {$[0.175,0.815]$} & $-0.429^{*}$ & {$[-0.760,0.037]$} \\
\hline
\end{tabular}

Cerebellar peduncle FA

\begin{tabular}{lllllll}
\hline i-SCP & 0.268 & {$[-0.173,0.655]$} & 0.255 & {$[-0.120,0.623]$} & -0.281 & {$[-0.661,0.167]$} \\
\hline C-SCP & 0.329 & {$[-0.108,0.732]$} & 0.339 & {$[0.021,0.671]$} & -0.325 & {$[-0.742,0.125]$} \\
\hline i-MCP & 0.129 & {$[-0.200,0.497]$} & 0.144 & {$[-0.248,0.546]$} & -0.116 & {$[-0.465,0.240]$} \\
\hline C-MCP & 0.109 & {$[-0.350,0.505]$} & -0.008 & {$[-0.490,0.524]$} & -0.103 & {$[-0.509,0.430]$} \\
\hline i-ICP & 0.252 & {$[-0.168,0.615]$} & 0.337 & {$[-0.014,0.670]$} & -0.271 & {$[-0.632,0.147]$} \\
\hline C-ICP & 0.018 & {$[-0.447,0.509]$} & 0.050 & {$[-0.404,0.582]$} & -0.076 & {$[-0.543,0.405]$}
\end{tabular}

* indicates significant correlation at $p<0.05$ (2-tailed) after bootstrap with 1000 samples. Abbreviations: ipsilesional (i-) and contralesional (c-) ROIs: superior corona radiata (SCR), posterior limb of the internal capsule (PLIC), and cerebral peduncle (CP), superior (SCP), middle (MCP) and inferior (ICP) cerebellar 
peduncles, superior longitudinal fasciculus (SLF), genu and body of the corpus callosum (CC), anterior corona radiata (ACR), anterior limb of the internal capsular (ALIC),

Table 3. Fixed effects of each behavioral score LMM over time, with FA measures from motor-related tracts as factors and accounting for cell therapy and demographics.

\begin{tabular}{|c|c|c|c|}
\hline Factors & dof & $F$ & $p$-value \\
\hline \multicolumn{4}{|c|}{ PPT (AIC=251; residuals: normal distribution) } \\
\hline Intercept & $(1,33.548)$ & 32.181 & 0.000 \\
\hline Treatment & $(1,21.375)$ & 0.566 & 0.460 \\
\hline Sex & $(1,17.244)$ & 0.028 & 0.868 \\
\hline Age & $(1,23.159$ & 0.795 & 0.382 \\
\hline i-PLIC by session & $(3,42.288)$ & 4.974 & 0.005 \\
\hline i-SLF by session & $(3,44.216)$ & 4.048 & 0.013 \\
\hline c-ALIC by session & $(3,42.397)$ & 8.169 & $<0.001$ \\
\hline Lesion Volume & $(1,27.972)$ & 10.117 & 0.004 \\
\hline \multicolumn{4}{|c|}{ Grip (AIC=376.85; residuals: normal distribution) } \\
\hline Intercept & $(1,33.170)$ & 17.436 & 0.000 \\
\hline Cell-therapy & $(1,20.933)$ & 1.760 & 0.199 \\
\hline Sex & $(1,19.140)$ & 8.011 & 0.011 \\
\hline Age & $(1,22.520)$ & 2.247 & 0.148 \\
\hline i-CP by session & $(3,37.230)$ & 3.427 & 0.027 \\
\hline c-ALIC by session & $(3,36.657)$ & 5.343 & 0.004 \\
\hline
\end{tabular}




\begin{tabular}{|lccc|}
\hline \multicolumn{4}{|l|}{ MST (AIC=589; residuals: normal } \\
\hline Intercept & $(1,28.064)$ & 0.548 & 0.465 \\
\hline Cell-therapy & $(1,22.874)$ & 0.837 & 0.370 \\
\hline Sex & $(1,22.533)$ & 0.005 & 0.943 \\
\hline Age & $(1,24.803)$ & 1.423 & 0.244 \\
\hline i-SLF by session & $(3,26.870)$ & 7.660 & 0.001 \\
\hline Body-CC by session & $(3,26.283)$ & 6.275 & 0.002 \\
\hline Lesion Volume & $(1,23.732)$ & 9.201 & 0.006 \\
\hline
\end{tabular}

$A / C$ indicates Akaike Information Criterion; dof, degrees of freedom; by, factor by session interaction, such as an effect of a tract at the first session. ROIs: ipsilesional (i-) and contralesional (c-) posterior limb of the internal capsule (PLIC), and cerebral peduncle (CP), superior longitudinal fasciculus (SLF), body of the corpus callosum (CC), anterior corona radiata (ACR). See Tables S4-S6 for more details.

\section{Figures}




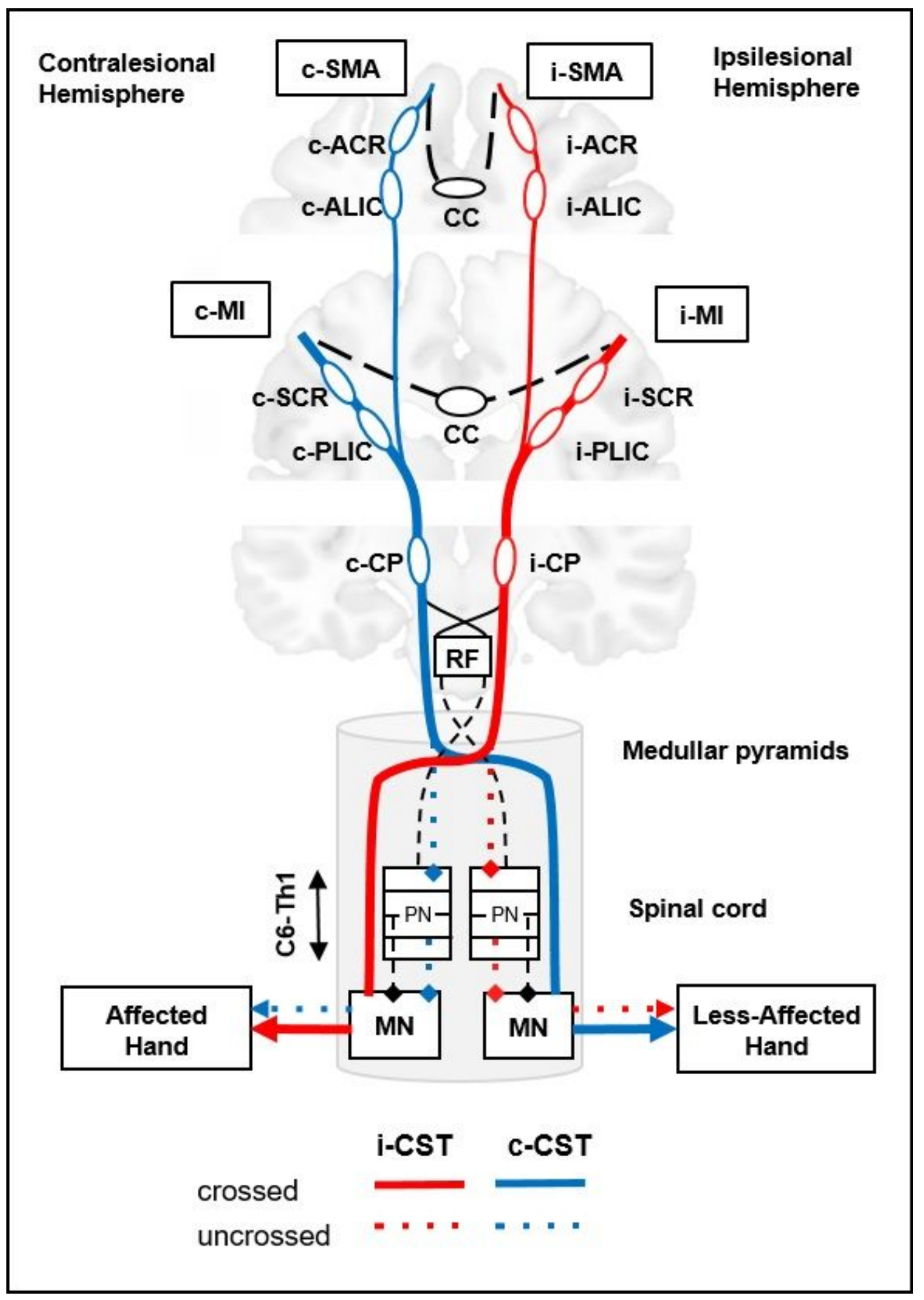

\section{Figure 1}

Schematic representation of crossed and uncrossed fibers of the corticospinal tract (CST). ACR and ALIC fibers emerging from the SMA and PMC and SCR-PLIC fibers emerging from PMC and MI merge in the CP to form the CST, which continues in the pons and medulla. Then the CST is divided into 2 parts. 1) Crossed CST (solid red and blue lines): most CST fibers decussate in the medullar pyramids to descend in the contralateral spinal cord and terminate in the contralateral anterior spinal horn to distal extremity 
muscles (direct cortico-motoneurons) 2) Uncrossed CST (dotted red and blue lines): a small proportion of the CST descends in the ipsilateral spinal lateral funiculus without decussating and terminate bilaterally in the ventromedial intermediate zone to propriospinal neurons. In addition, information is shared between the ipsilesional and contralesional hemispheres through transcallosal fibers (CC, dashed dark lines), before travelling through the CST. Abbreviations: $\mathrm{CST}=$ corticospinal tract, $\mathrm{SCR}=$ Superior Corona Radiata; $\mathrm{PLIC}=$ posterior limb of internal capsule, $\mathrm{CP}=$ cerebral peduncle. Other $\mathrm{ROI}$ are $\mathrm{ACR}=$ Anterior Corona Radiata; $\mathrm{ALIC}=$ anterior limb of internal capsule; $\mathrm{CC}=$ Corpus Callosum; RF reticular formation; $\mathrm{PN}=$ propriospinal neurons; $\mathrm{MN}=$ motoneurons; $\mathrm{i}=$ ipsilesional and $\mathrm{c}=$ contralesional tracts.

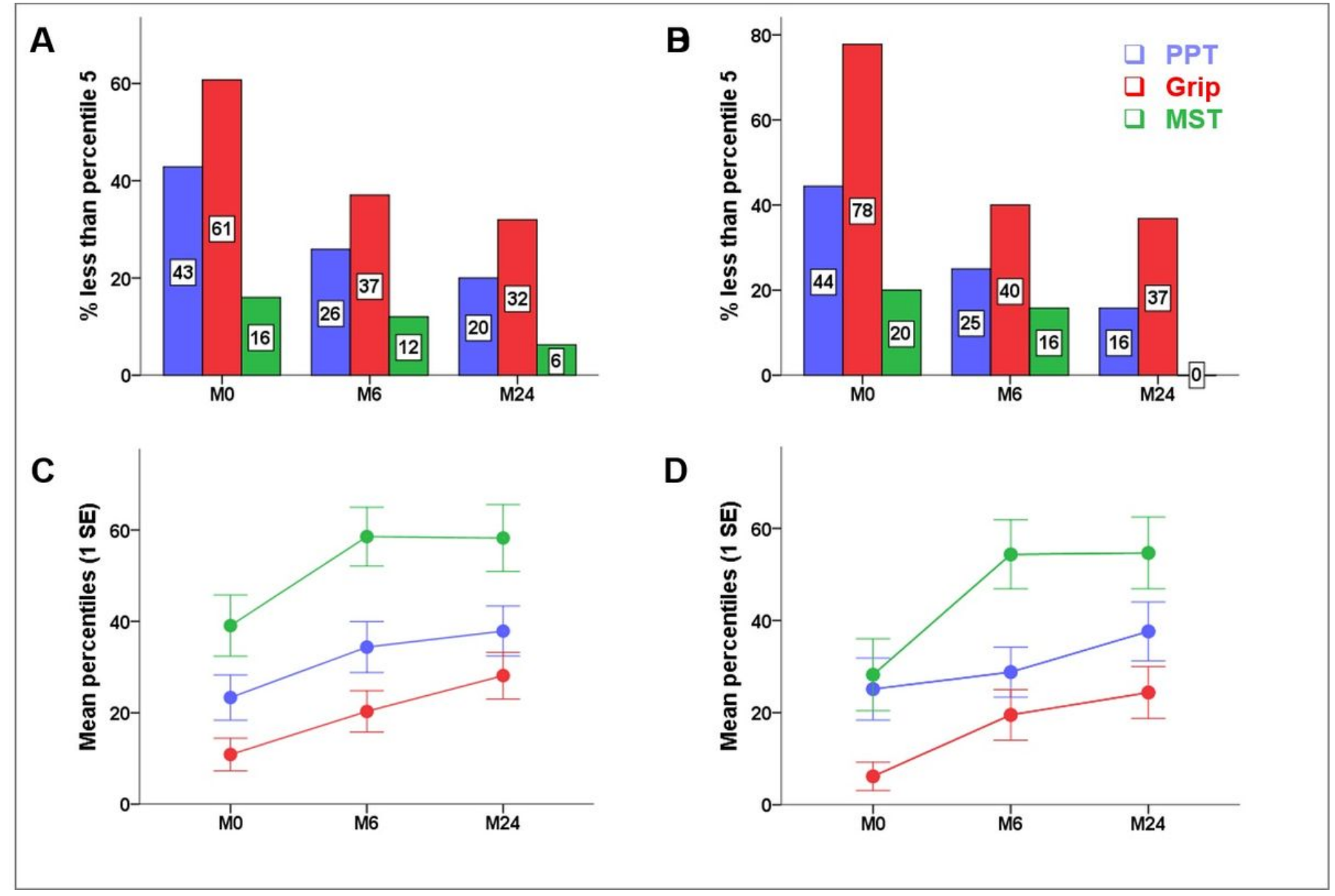

\section{Figure 2}

LAH impairment in the patients. A-Behavioral score frequency over time in the 28 patients. B- Behavioral score frequency over time in the 20 patients without cognitive deficit. C. Mean percentiles over time in the 28 patients. D. Mean percentiles over time in the patients without cognitive deficit.

\section{Supplementary Files}

This is a list of supplementary files associated with this preprint. Click to download.

- BIBChecklistAJ.docx 
- Strobe.pdf

- SupplementaryHannanu.pdf 